\title{
Knit Products of Some Groups and Their Applications
}

\author{
Firat Ateș (*) - A. Sinan Çevik (**)
}

\begin{abstract}
Let $G$ be a group with subgroups $A$ and $K$ (not necessarily normal) such that $G=A K$ and $A \cap K=\{1\}$. Then $G$ is isomorphic to the knit product, that is, the "two-sided semidirect product" of $K$ by $A$. We note that knit products coincide with Zappa-Szep products (see [18]).

In this paper, as an application of [2, Lemma 3.16], we first define a presentation for the knit product $G$ where $A$ and $K$ are finite cyclic subgroups. Then we give an example of this presentation by considering the (extended) Hecke groups. After that, by defining the Schur multiplier of $G$, we present sufficient conditions for the presentation of $G$ to be efficient. In the final part of this paper, by examining the knit product of a free group of rank $n$ by an infinite cyclic group, we give necessary and sufficient conditions for this special knit product to be subgroup separable.
\end{abstract}

\section{Introduction.}

The structure of semidirect products is well known. In fact the semidirect product of any two groups is a generalization of the direct product of these two groups which requires at least one of the factors to be normal in the product. In another words, if a group $G$ is a product $A B$ of two subgroups with $A$ normal and $A \cap B=\{1\}$ then conjugation of $A$ by the elements of $B$ gives an action of $B$ on $A$ by automorphisms. Moreover, if $A$ and $B$ are groups not known to be subgroups of another group and there is an action of $B$ on $A$ by automorphisms then a group structure (the semidirect product) on the set $A \times B$ can be defined so that conjugation of $A \times\{1\}$ by elements of $\{1\} \times B$ mirrors the given action.

(*) Indirizzo dell'A.: Department of Mathematics, Faculty of Science and Art, Balikesir University, Campus, 10145, Balikesir, Turkey.

E-mail: firat@balikesir.edu.tr

(**) Indirizzo dell'A.: Department of Mathematics, Faculty of Science, Selçuk University Campus, 42003 Konya, Turkey.

E-mail: sinan.cevik@selcuk.edu.tr

2000 Mathematics Subject Classification: 20E22, 20F05, 20F55, $20 \mathrm{~F} 32$. 
The next step along this path is the Zappa-Szep product of any two groups, which requires neither of the factors to be normal in the product. In other words, if the subgroup $A$ is not assumed to be normal then a similar situation exists. We first look at groups to get the main parts. Let $G$ be a group with identity $\{1\}$, subgroups $A, K$ satisfying $K \cap A=\{1\}$ and $G=A K$. Then each $g \in G$ is uniquely expressible as $g=a k$ with $k \in K$ and $a \in A$. We are now in a position to reserve certain products with $a \in A$ and $k \in K$ by considering $a k \in G$. We must have unique elements $k^{\prime} \in K$ and $a^{\prime} \in A$ such that $k a=a^{\prime} k^{\prime}$. This defines two functions

$$
(k, a) \longmapsto k^{a} \in K, \quad(k, a) \longmapsto k . a \in A
$$

(in fact these were called the mutual actions defined by the multiplication by Brin in [2]) that are unique subject to the relation

$$
k a=(k \cdot a)\left(k^{a}\right),
$$

for all $k \in K$ and $a \in A$. We remark that the details of the above material can be found in [2]. We also note that the terminology Zappa-Szep product was developed and suggested by G. Zappa in [20].

Moreover, in [14], it is proved that if a Lie algebra is the direct sum of two sub Lie algebras then one can write the bracket in a way that mimics semidirect products on both sides. This construction is called the knit product of graded Lie algebras. Additionally, in [14], the behaviour of homomorphisms with respect to knit products was investigated. The integrated version of a knit product of Lie algebras will be called the knit product of groups which coincides with the Zappa-Szep product (see [18]).

Throughout this paper the notation $Z_{n}$ denotes the cyclic group of order $n, D_{n}$ denotes the dihedral group of order $2 n$ and $S_{n}$ denotes the symetric group of order $n$ !, where $n \in \mathbb{N}$.

\section{Preliminaries.}

In this section we define the knit product of any two groups by using the action given (1) and then give a standard presentation for this product. We note that material similar to this section may also be found in [2] and [14].

Let $A$ and $K$ be subgroups of a group $G$ as defined in the previous section.

Lemma 2.1. The knitted pair of actions $(\tilde{\alpha}, \tilde{\beta})$ for $(A, K)$ are mappings

$$
\begin{array}{cl}
\tilde{\alpha}: K \times A \longrightarrow A, & \tilde{\beta}: K \times A \longrightarrow K \\
(k, a) \longmapsto k . a, & (k, a) \longmapsto k^{a}
\end{array}
$$


such that

i) $\alpha: K \longrightarrow A u t(A)$ is a group homomorphism, so $\alpha_{k_{1}}\left(\alpha_{k_{2}}\right)=\alpha_{k_{1} k_{2}}$ and $\alpha_{1}=I d_{A}$, where $\alpha_{k}(a):=\tilde{\alpha}(k, a)$,

ii) $\beta: A \longrightarrow A u t(K)$ is a group anti homomorphism, in other words, $\beta_{a_{1}}\left(\beta_{a_{2}}\right)=\beta_{a_{2} a_{1}}$ and $\beta_{1}=I d_{K}$, where $\beta_{a}(k):=\tilde{\beta}(k, a)$,

iii) $\alpha_{k}\left(a_{1} a_{2}\right)=\alpha_{k}\left(a_{1}\right) \cdot \alpha_{\beta_{a_{1}}(k)}\left(a_{2}\right)$,

iv) $\beta_{a}\left(k_{1} k_{2}\right)=\beta_{\alpha_{k_{2}}(a)}\left(k_{1}\right) \cdot \beta_{a}\left(k_{2}\right)$, for all $a, a_{1}, a_{2} \in A, k, k_{1}, k_{2} \in K$.

One may find the proof of the above lemma in [2, Lemma 3.2] or [14]. Moreover, by considering the actions given in (1), knit products of groups can be defined as follows.

Definition 2.2. Let $A$ and $K$ be groups, and let $\alpha, \beta$ be homomorphisms defined by

$$
\beta: A \longrightarrow A u t(K), a \longmapsto \beta_{a} \text { and } \alpha: K \longrightarrow A u t(A), k \longmapsto \alpha_{k}
$$

respectively, forall $a \in A$ and $k \in K$. Then the "knit product" $G=A \bowtie_{(\alpha, \beta)} K$ of $K$ by $A$ is defined on the set $A \times K$ by the following operation:

$$
\left(a_{1}, k_{1}\right)\left(a_{2}, k_{2}\right)=\left(a_{1} \alpha_{k_{1}}\left(a_{2}\right), \beta_{a_{2}}\left(k_{1}\right) k_{2}\right) .
$$

The identity is $(1,1)$ and the inverse of an element $(a, k)$ is

$$
(a, k)^{-1}=\left(\alpha_{k^{-1}}\left(a^{-1}\right), \beta_{a^{-1}}\left(k^{-1}\right)\right) .
$$

In fact $A \times\{1\}$ and $\{1\} \times K$ are subgroups of $A \bowtie_{(\alpha, \beta)} K$ which are isomorphic to $A$ and $K$, respectively.

Similar definitions of knit product can also be found in [2], [14] and [18]. (We note that if $A$ and $K$ are topological groups or Lie groups and $\tilde{\alpha}, \tilde{\beta}$ are continuous or smooth, then $A \bowtie_{(\alpha, \beta)} K$ is also a topological group or Lie group, respectively, which will not be needed in this paper).

REMARK 2.3. 1) In Definition 2.2, if $\alpha \equiv I d_{A}$ (or $\left.\beta \equiv I d_{B}\right)$ then the knit product $A \bowtie_{(\alpha, \beta)} K$ becomes the semidirect product.

2) We know that the semidirect product of any two groups is actually equivalent to the split extension. Also, by the previous material, the knit product can be thought as a two-sided semidirect product by assuming the factors not to be normal. Therefore knit products can also be regarded as a type of split extension. To show this fact, it is enough to generalize Propositions 2.1 and 2.3 given in [3, Chapter IV].

One can find an earlier proof for the following result in [14]. 
Proposition 2.4. Let $G$ be a group and $A, K$ be subgroups of $G$. Suppose that $G=A K$ and $A \cap K=\{1\}$. Then $G \cong A \bowtie_{(\alpha, \beta)} K$.

Proof. Let $k \cdot a=\tilde{\alpha}(k, a) \cdot \tilde{\beta}(k, a)$ be the unique decomposition of $k \cdot a$ in $G=A K$. Then

$$
a_{1} k_{1} a_{2} k_{2}=a_{1} \tilde{\alpha}\left(k_{1}, a_{2}\right) \tilde{\beta}\left(k_{1}, a_{2}\right) k_{2}=\left(a_{1} \alpha_{k_{1}}\left(a_{2}\right)\right) .\left(\beta_{a_{2}}\left(k_{1}\right) k_{2}\right) .
$$

Thus we just need to show that the knitted pair of actions $(\tilde{\alpha}, \tilde{\beta})$ satisfies the four conditions of Lemma 2.1. It is clear that we have $\tilde{\alpha}(1, a)=a, \tilde{\beta}(1, a)=1$, $\tilde{\alpha}(k, 1)=1, \tilde{\beta}(k, 1)=k$. In fact comparing coefficients in the law of associativity of $G$ gives two equations. Then setting suitable elements in these equations to 1 gives all conditions of Lemma 2.1.

In [2], Brin defined a standard presentation for the knit product of groups (and monoids as well) by stating a similar version of the following lemma.

Lemma 2.5 [2, Lemma 3.16]. Suppose that $\mathcal{P}_{A}=\langle X ; R\rangle$ and $\mathcal{P}_{K}=$ $=\langle Y ; S\rangle$ are presentations for the groups $A$ and $K$, respectively under the maps $y \longmapsto k_{y}(y \in Y), x \longmapsto a_{x}(x \in X)$ with $X \cap Y=\emptyset$. Then a presentation for the structure defined by (3) on $A \times K$ is

$$
\mathcal{P}=\langle X, Y ; R, S, T\rangle
$$

in which $T$ consists of all pairs $\left(y x,(y \cdot x)\left(y^{x}\right)\right)$, as given in (2), for $(y, x) \in K \times A$.

\section{The knit product of cyclic groups.}

In this section, as an application of Lemma 2.5, we will define a presentation (see Proposition 3.1 below) for the knit product of finite cyclic groups in terms of the generators and relators of these groups. After that, by taking $A$ an infinite group and $K=Z_{p}$ ( $p$ a prime), we will give an example of the knit product for the infinite case.

Let $A$ and $K$ be both $Z_{n}$ and $Z_{m}$ generated by $x$ and $y$, respectively, and let $G$ be the knit product $A \bowtie_{(\alpha, \beta)} K$, where $\alpha: K \longrightarrow A u t(A)$ and $\beta: A \longrightarrow A u t(K)$. We then have

Proposition 3.1. Let $\mathcal{P}_{A}=\left\langle x ; x^{n}\right\rangle$ and $\mathcal{P}_{K}=\left\langle y ; y^{m}\right\rangle$ be presentations for the groups $A$ and $K$, respectively. Suppose that $x^{t^{m}-1}=1_{A}$ and 
$y^{l^{n}-1}=1_{B}(1 \leq|t|<n, 1 \leq|l|<m)$. Then $G$ has a presentation

$$
\mathcal{P}_{G}=\left\langle x, y ; x^{n}, y^{m}, T_{y x}\right\rangle,
$$

where $T_{y x}$ consists of all pairs $\left(y x, x^{t} y^{l}\right)$.

Proof. Let $\delta_{t}(1 \leq|t|<n)$ and $\psi_{l}(1 \leq|l|<m)$ be automorphisms of $A$ and $K$, respectively. Assume that $x^{t^{m}}=x$ and $y^{l^{n}}=y$. Then we have mappings $y \longrightarrow A u t(A)$ and $x \longrightarrow A u t(K)$. By [7], these induce homomorphisms

$$
\begin{aligned}
& \alpha: K \longrightarrow \operatorname{Aut}(A) \text { and } \beta: A \longrightarrow \operatorname{Aut}(K) \\
& y \longmapsto \delta_{t}, \quad x \longmapsto \psi_{l}
\end{aligned}
$$

if and only if

$$
\delta_{t}^{m}=i d_{A} \quad \text { and } \quad \psi_{l}^{n}=i d_{B}
$$

By the assumption on the generator $x$, the homomorphisms $\delta_{t}^{m}$ and $i d_{A}$ are equal if and only if $[x] \delta_{t}^{m}=[x] i d_{A}$. Similarly, by the assumption on $y, \psi_{l}^{n}$ and $i d_{B}$ are equal if and only if $[y] \psi_{l}^{n}=[y] i d_{B}$. These imply that $y x=x^{t} y^{l}$. Hence, by Lemma 2.5, we obtain the presentation $\mathcal{P}_{G}$ in (5) for the group $Z_{n} \bowtie(\alpha, \beta) Z_{m}$.

ExAmple 3.2. For any prime p, let us determine $Z_{2} \bowtie_{(\alpha, \beta)} Z_{\text {sp }}$. So suppose that $\left\langle x ; x^{2}\right\rangle$ and $\left\langle y ; y^{p}\right\rangle$ are the presentations for $Z_{2}$ and $Z_{p}$, respectively. Then the homomorphisms $\beta: Z_{2} \longrightarrow A u t\left(Z_{p}\right) \cong Z_{p-1}$ and $\alpha: Z_{p} \longrightarrow$ Aut $\left(Z_{2}\right)$ are defined by $x \longmapsto \beta_{x}: y \longmapsto y^{-1}$ and $y \longmapsto \alpha_{y}: x \longmapsto x^{-1}$, respectively. Hence, by Proposition 3.1, we have a presentation $\left\langle x, y ; x^{2}, y^{p}, y x=x^{-1} y^{-1}\right\rangle$ for the group $Z_{2} \bowtie_{(\alpha, \beta)} Z_{p}$. In fact this presentation can be written as $\left\langle x, y ; x^{2}, y^{p},(y x)^{2}\right\rangle$, and so this implies that $Z_{2} \bowtie_{(\alpha, \beta)} Z_{p} \cong D_{p}$. In particular, if we take $p=2$ then we obtain $Z_{2} \bowtie_{(\alpha, \beta)} Z_{3} \cong D_{3} \cong S_{3}$.

Actually if we choose any positive integer $m$ instead of $p$ in the above calculations then we obtain $Z_{2} \bowtie_{(\alpha, \beta)} Z_{m} \cong D_{m}$, where $m \geq 2$.

The following example, obtained by considering the (extended) Hecke groups, is an application about infinite case for the presentation $\mathcal{P}$, defined in Lemma 2.5. We note that the fundamental material about (extended) Hecke groups can be found, for instance, in [11, 17]. Recall that Hecke groups $H\left(\lambda_{q}\right)$ are presented by $\left\langle x, y ; x^{2}, y^{q}\right\rangle$ while extended Hecke groups $\bar{H}\left(\lambda_{q}\right)$ are presented by $\left\langle x, y, r ; x^{2}, y^{q}, r^{2},(x r)^{2},(y r)^{2}\right\rangle$. In fact $\bar{H}\left(\lambda_{q}\right) \cong$ $\cong D_{2} * z_{2} D_{q}$. 
EXAMPLE 3.3. Let $A$ be the Hecke group $H\left(\lambda_{q}\right)$, where $q \geq 3$ and $q \in Z^{+}$. Also let $K$ be the group $Z_{p}$ generated by $r$, where $p$ is a prime. Let us consider the homomorphisms

$$
\begin{array}{rlccccc}
\alpha: Z_{p} & \longrightarrow & H\left(\lambda_{q}\right) & \text { and } & \beta \quad: H\left(\lambda_{q}\right) & \longrightarrow & Z_{p} \\
r & \longmapsto \alpha_{r}(x)=x^{-1} & & x & \longmapsto \beta_{x}(r)=r^{t} \\
\alpha_{r}(y)=y^{j} & & & y & \longmapsto \beta_{y}(r)=r^{s}
\end{array}
$$

where $1 \leq t, s<p, 1 \leq j<q$ such that $x^{-1^{p}}=x, y^{j^{p}}=y, r^{t^{2}}=r$ and $r^{s^{q}}=r$. We then get the relators

$$
r y=\alpha_{r}(y) \beta_{y}(r)=y^{j} r^{s} \quad \text { and } \quad r x=\alpha_{r}(x) \beta_{x}(r)=x^{-1} r^{t} .
$$

Hence, by Lemma 2.5,

$$
\mathcal{P}=\left\langle x, y, r ; x^{2}, y^{q}, r^{p}, r y=y^{j} r^{s}, r x=x^{-1} r^{t}\right\rangle
$$

is a presentation for the group $H\left(\lambda_{q}\right) \bowtie_{(\alpha, \beta)} Z_{p}$.

In presentation (6), we can choose $p=2, s=1$ and $j=t=-1$ since we have $x^{-1^{2}}=x, y^{-1^{2}}=y, r^{1}=r$ and $r^{-1^{2}}=r$. Therefore

$$
H\left(\lambda_{q}\right) \bowtie_{(\alpha, \beta)} Z_{2} \cong \bar{H}\left(\lambda_{q}\right) \cong D_{2} * Z_{2} D_{q} .
$$

\section{1 - The Schur multiplier.}

In this part of the section we define the Schur multiplier (or, equivalently, the second homology group) of the knit product of two finite cyclic groups.

Let $K$ be a cyclic group of order $m$ with a presentation $\mathcal{P}_{K}=\left\langle y ; y^{m}\right\rangle$, and let $A$ be cyclic group of order $p$ ( $p$ is a prime) with a presentation $\mathcal{P}_{A}=\left\langle x ; x^{p}\right\rangle$. Then, by Proposition 3.1, a presentation for $G=A \bowtie_{(\alpha, \beta)} K$ is given by

$$
\mathcal{P}=\left\langle x, y ; x^{p}, y^{m}, y x=x^{t} y^{l}\right\rangle,
$$

where $x^{t^{m}-1}=1$ and $y^{l^{p}-1}=1$ such that $1 \leq|t|<n$ and $1 \leq|l|<m$. Suppose that

- $(l-1, m d)=d$ with $d=(l-1, m)$ and

- $l^{p} \equiv 1(\bmod m d)$.

We then have the following result. 
THEOREM 3.4. Let $G$ be the knit product with presentation $\mathcal{P}$ as in (7). Then

$$
H_{2}(G) \cong \begin{cases}\{1\} & \text { if } d=1 \\ Z_{p} & \text { if } d=p .\end{cases}
$$

Before giving a proof of this result, we should note that the equivalence class containing a factor set $\gamma$ of $G$ will be denoted by $\{\gamma\}$. Let $M$ be an algebraically closed field of characteristic zero with its multiplicative group $M^{*}=M-\{0\}$. The set $H_{2}\left(G, M^{*}\right)$ (which we denote it by $H_{2}(G)$ ) of all equivalence classes of factor sets of $G$ over $M$ forms an abelian group under the multiplication defined by $\left\{\gamma_{1}\right\}\left\{\gamma_{2}\right\}=\left\{\gamma_{1} \gamma_{2}\right\}$. This group is called the Schur multiplier of $G$, and it is actually the second homology group where $M^{*}$ is a trivial $G$-module. We refer the reader to [12] for the details and applications of the Schur multiplier.

Proof. Suppose that $d=1$. Then $p$ does not divide $m$ and hence every Sylow subgroups of $G$ is cyclic, that is $H_{2}(G)=\{1\}$.

Now assume that $d=p$. In that case we will show that $H_{2}(G)$ is a cyclic group of order $p$ with

$$
G_{1}=\left\langle y_{1}, x_{1} ; y_{1}^{m p}, x_{1}^{p},\left(x_{1}^{t}\right)^{-1} y_{1} x_{1}=y_{1}^{l}\right\rangle
$$

is a representation group of $G$. Since $G$ is generated by two elements and has three relators, by [15], $H_{2}(G)$ must be cyclic. On the other hand, $G$ has a subgroup $\langle x\rangle$ of index $p$. Hence $\left|H_{2}(G)\right| \leq p$. Moreover, by the assumption, since $p \mid m$ and $y^{l^{p}-1}=1$ then $(l, m)=1$. It follows that $(l, m p)=1$. Then we have $l^{p} \equiv 1(\bmod m p)$. Hence $G_{1}$ is a well-defined group. By [15, Proposition 2.2], we have

$$
\left[G_{1}, G_{1}\right]=\left\langle y_{1}^{l-1}\right\rangle=\left\langle y_{1}^{p}\right\rangle \quad \text { and } \quad Z\left(G_{1}\right)=\left\langle y_{1}^{m}\right\rangle .
$$

Therefore $\left\langle y_{1}^{m}\right\rangle=\left[G_{1}, G_{1}\right] \cap Z\left(G_{1}\right)$ and, by the isomorphism theorem, we obtain $G_{1} /\left\langle y_{1}^{m}\right\rangle \cong G$. Hence it follows that $\left|H_{2}(G)\right| \geq p$. Therefore, $H_{2}(G)$ is cyclic of order $p$, and $G_{1}$ is a representation group of $G$.

\section{$3.2-$ Efficiency.}

In this part, let us recall the definition of efficiency on groups and then give a result about efficiency for the knit product of finite cyclic groups by considering Theorem 3.4 . 
Let $G$ be a finitely presented group, and let $\mathcal{P}=\langle\mathbf{x} ; \mathbf{r}\rangle$ be a finite presentation for $G$. Then the Euler characteristic of $\mathcal{P}$ is defined by $\chi(\mathcal{P})=1-|\mathbf{x}|+|\mathbf{r}|$, where $|$.$| denotes the number of elements in the set.$ Let $\delta(G)=1-r k_{Z}\left(H_{1}(G)\right)+d\left(H_{2}(G)\right)$ where $r k_{Z}($.$) denotes the Z$-rank of the torsion-free part and $d($.$) means the minimal number of generators.$ Then, by [9], for the presentation $\mathcal{P}$, it is always true that $\chi(\mathcal{P}) \geq \delta(G)$. We then define $\chi(G)=\min \{\chi(\mathcal{P}): \mathcal{P}$ is a finite presentation for $G\}$. In view of these facts, for a group $G$, we say that a presentation $\mathcal{P}_{0}$ for $G$ is called minimal if $\chi\left(\mathcal{P}_{0}\right) \leq \chi(\mathcal{P})$, for all presentations $\mathcal{P}$ of $G$ and a presentation $\mathcal{P}_{0}$ is called efficient if $\chi\left(\mathcal{P}_{0}\right)=\delta(G)$.

A brief survey of known results on efficiency can be found in [6]. In fact there is interest not only in finding efficient presentations, but also finding presentations which are efficient on the minimal number of generators (see [19]).

Let $d=p$ in Theorem 3.4. Then we have the following result.

THEOREM 3.5. Let $G=Z_{p} \bowtie_{(\alpha, \beta)} Z_{m}$ with a presentation $\mathcal{P}$ as in (7). Suppose that $(l-1, m p)=p$ with $p=(l-1, m)$ and $l^{p} \equiv 1(\bmod m p)$. Then $\mathcal{P}$ is an efficient presentation on 2-generators for the group $G$.

Proof. First of all let us consider the lower bound $\delta(G)$ of $G$. Since the order of $G$ is $m p$ (by considering Remark 2.3-2)), we get $r k_{\mathrm{Z}}\left(H_{1}(G)\right)=0$. Thus we have $\delta(G)=1+d\left(H_{2}(G)\right)$. Actually, by Theorem $3.4, H_{2}(G) \cong Z_{p}$ and so $d\left(H_{2}(G)\right)=1$. Hence $\delta(G)=2$. In the second part of the proof, a simple calculation shows that the Euler characteristic of $\mathcal{P}$ is equal to 2 . Hence $\delta(G)=\chi(\mathcal{P})$ and so $\mathcal{P}$ is an efficient presentation. Moreover, since $G$ is a 2-generator group, we cannot apply any reduction on the generating set of $\mathcal{P}$, which means this efficient presentation $\mathcal{P}$ must have 2 generators, as required.

As an application of Theorem 3.5, let us choose $t=1, l=-1$ and $p=2$. Then we get the dihedral group $D_{m}$ of order $2 m(m \geq 2)$ presented by

$$
\mathcal{P}=\left\langle x, y ; x^{2}, y^{m},(y x)^{2}\right\rangle
$$

Thus a straightforward computation shows that

COROLlary 3.6. $\quad \mathcal{P}$, as in (8), is an efficient presentation for the group $D_{m}$ if $m$ is even integer greater than or equal to 4. 
REMARK 3.7. In Theorem 3.5, if $d=1$ then $\mathrm{H}_{2}(G)$ is trivial (by Theorem 3.4), and so $d\left(H_{2}(G)\right)=0$. Thus this gives the inefficiency of the presentation $\mathcal{P}$. Therefore it might be useful to study whether or not the presentation $\mathcal{P}$ in $(7)$ is minimal when $d=1$.

REMARK 3.8. In Example 3.3, up to isomorphism, we obtained a presentation for the extended Hecke group $\bar{H}\left(\lambda_{q}\right)$. In fact the efficiency of $\bar{H}\left(\lambda_{q}\right)$ has been examined under certain conditions in [8].

\section{Subgroup separability of $\boldsymbol{F} \bowtie_{(a, \beta)} Z$.}

In [10, Problem 5], Scott asked a question which was whether all semidirect products $F \bowtie_{\alpha} Z_{\text {with }} \alpha \in A u t(F)$ are subgroup separable. We should note that the first negative answer was given by Burns, Karras and Solitar in [4]. After that it has been positively answered by Metaftsis and Raptis [13, Corollary 3].

Now our aim is to lift the problem above to knit products. Thus, by considering the knit product of a free group of rank $n$ by infinite cyclic group, we give a partial answer to it.

By using the following lemma, we will give necessary and sufficient conditions for the knit product $F \bowtie_{(\alpha, \beta)} Z_{\text {Z }}$ to be subgroup separable. We recall that a group $G$ is said to be subgroup separable if, for every finitely generated subgroup $H$ of $G, H$ is the intersection of finite index subgroup of $G$.

LEMma 4.1 ([13)]. Let $K$ be a finitely generated abelian group and let $A$, $B$ be subgroups of $K$ such that $G$ is the $H N N$-extension

$$
G=\left\langle t, K ; t^{-1} A t=B\right\rangle .
$$

Then $G$ is subgroup separable if and only if $A \cap B$ is a subgroup of finite index in both $A$ and $B$, and there is a finitely generated normal subgroup of $G$, say $H$, such that $H$ has finite index in $A \cap B$.

Before giving our theorem, let us give the definition of right layered basis which will be needed for our result. Suppose that $\alpha$ is an automorphism of the free group $F$ having rank $n$ and $X=\left\{x_{1}, x_{2}, \cdots, x_{n}\right\}$ is a basis for $F$. Then $X$ is a right layered basis for $\alpha$ if

$$
\alpha\left(x_{1}\right)=x_{1} \quad \text { and } \quad \alpha\left(x_{i}\right)=x_{i} w_{i},
$$


where $w_{i} \in F\left(x_{1}, \cdots, x_{i-1}\right)$ for $2 \leq i \leq n$. The existence of a right layered basis for an automorphism of free groups with rank $n$ has been shown in [5].

Let us consider $G=F \bowtie_{(\alpha, \beta)} \mathrm{Z}$. We then have the following result.

THeOREm 4.2. $G$ is subgroup separable if and only if $\alpha$ is the identity automorphism.

Proof. Let us suppose the set $Z$ is generated by $y$. Also let us assume $1 \neq \alpha \in A u t(F)$ such that there exists a right layered basis $X=\left\{x_{1}, x_{2}, \cdots, x_{n}\right\}$ for $\alpha$ as in (9). Therefore, by Lemma 2.5, one can easily show that $G$ has a presentation

$$
G=\left\langle x_{1}, x_{2}, \cdots, x_{n}, y ; y x_{1}=x_{1} y^{j_{1}}, y x_{2}=x_{2} w_{2} y^{j_{2}}, \cdots, y x_{n}=x_{n} w_{n} y^{j_{n}}\right\rangle,
$$

where $w_{i}$ 's are words as in (9) and each $j_{i}(1 \leq i \leq n)$ is an integer. Since $\alpha \neq 1$, at least one element of the $w_{i}$ 's is a non-trivial word. Let $w_{s}$ be the first such a word, where $w_{s} \in F\left(x_{1}, x_{2}, \cdots, x_{s-1}\right)$. Then we can choose $w_{s}=x_{m_{1}} x_{m_{2}} \cdots x_{m_{s-1}}$ such that $x_{m_{1}}, x_{m_{2}}, \cdots, x_{m_{s-1}} \in\left\{x_{1}, x_{2}, \cdots, x_{s-1}\right\}$. Also let $H$ be a subgroup of $G$ generated by $\left\{x_{s}, w_{s}, y\right\}$. Then $H$ has a presentation of the form

$$
\begin{gathered}
H=\left\langle x_{s}, w_{s}, y ; y w_{s} y^{-j_{i_{1}}}=x_{m_{1}} y^{j_{i_{1}}-1} x_{m_{2}} y^{j_{i_{1}}-1} \cdots x_{m_{s-1}} y^{j_{i_{1}}-1},\right. \\
\left.y x_{s} y^{-j_{i_{2}}}=x_{s} w_{s} \quad\left(1 \leq j_{i_{1}}, j_{i_{2}} \leq n\right)\right\rangle
\end{gathered}
$$

since $y x_{m_{k}} y^{-j_{i_{1}}}=x_{m_{k}}$ for all $1 \leq m_{k} \leq s-1$. In this step, let us take $j_{i_{1}}=1=j_{i_{2}}$. Then the above presentation can be rewritten as follows:

$$
H=\left\langle x_{s}, w_{s}, y ; y w_{s} y^{-1}=w_{s}, x_{s}^{-1} y x_{s}=y w_{s}\right\rangle .
$$

This means $H$ is an $\mathrm{HNN}$-extension with base group $K$ that is a free abelian group of rank two generated by $\left\{w_{s}, y\right\}$ and stable letter $x_{s}$. Let the isomorphic subgroups of $K$ be $C$ and $D$ generated by $\{y\}$ and $\left\{y w_{s}\right\}$, respectively. It is obvious that $C \cap D=\{1\}$ and, by Lemma $4.1, H$ is not subgroup separable. Therefore $G$ cannot be subgroup separable since it contains $H$ which gives a contradiction by the assumption $\alpha \neq 1$.

On the other hand, let $\alpha=1$. Then $G \cong Z_{\beta} F$ which is subgroup separable since it has index two subgroup isomorphic to $Z \times F$ which is subgroup separable. Thus $G$ is subgroup separable (by the results given in $[1,16])$, as required. 


\section{REFERENCES}

[1] R. B. J. T. Allenby - R. J. GRegorac, On locally extended residually finite groups in "Conference on Group Theory, University of Wisconsin 1972", Lecture Notes in Mathematics, Vol. 319 (Springer-Verlag, Berlin-New York, 1973), pp. 9-17.

[2] M. G. BRIN, On the Zappa-Szep product, Comm. Algebra 33 (2005), pp. 393-424.

[3] K. S. Brown, Cohomology of Groups, Springer-Verlag, 87, 1987.

[4] R. G. Burns - A. Karrass - D. Solitar, A note on groups with separable finitely generated subgroups, Bull. Aust. Math. Soc., 36 (1987), pp. 153-160.

[5] D. J. Collins - E. C. Turner, All automorphisms of free groups with maximal rank fixed subgroups, Math. Proc. Cambridge Philos. Soc., 119 (1996), pp. 615-630.

[6] A. S. CुEVIK, The efficiency of standard wreath products, Proc. of Edinburgh Math. Soc., 43 (2000), pp. 415-423.

[7] A. S. ÇEVIK, The p-Cockcroft property of semidirect products of monoids, Int. J. Alg. and Compt., 13, 1 (2003), pp. 1-16.

[8] A. S. ÇEVIK - N. Y. ÖZGÜR - R. SAHIN, The extended Hecke groups as a semidirect product and related results, Inter. J. of Applied Math. and Statistics, 13 (S08), (September 2008), pp. 63-72.

[9] D. B. A. Epstein, Finite presentations of groups and 3-manifolds, Quart. J. Math. Oxford Ser (2), 12 (1961), pp. 205-212.

[10] S. M. Gersten, Selected problems in Combinatorial Group Theory and Topology (S. M. Gersten and J. R. Stallings, Eds.), Annals Mathematics Studies, 111, Princeton Univ. Press, Princeton, 1987.

[11] S. Huang, Generalized Hecke groups and Hecke polygons, Ann. Acad. Sci. Fenn. Math., 24 (1999), pp. 187-214.

[12] G. Karpilovsky, The Schur Multiplier, LMS Monograms New Series 2, Oxford Science Publications, 1987.

[13] V. Metaftsis - E. RaPTIS, Subgroup separability of HNN-Extensions with abelian base group, Journal of Algebra, 245 (2001), pp. 42-49.

[14] P. W. Michor, Knit products of graded Lie algebras and groups, Suppl. Rendiconti Circolo Matematico di Palermo Ser. II, 22 (1989), pp. 171-175.

[15] S. A. PARK, Projective representations of some finite groups, J. Korean Math. Soc., 22 (1985), pp. 173-180.

[16] P. ScotT, Subgroups of surface groups are almost geometric, J. London Math. Soc., 17 (1978), pp. 555-565.

[17] R. SAHIN - S. IKIKARDES - O. KoRUOGLU, Some normal subgroups of the extended Hecke groups $\bar{H}\left(\lambda_{p}\right)$, Rocky Mountain J. Math., 36, 3 (2006), pp. 1033-1048.

[18] J. SzEP, On the structure of groups which can be represented as the product of two subgroups, Acta Sci. Math. Szeged, 12 (1950), pp. 57-61.

[19] J. W. WAMsLeY, Minimal presentations for finite groups, Bull. London Math. Soc., 5 (1973), pp. 129-144.

[20] G. ZAPPA, Sulla costruzione dei gruppi prodotto di due dati sottogruppi permutabili tra loro, Atti Secondo Congresso Un. Mat. Ital., Bologna, 1940, Edizioni Cremonense (Rome 1942), pp. 119-125.

Manoscritto pervenuto in redazione il 18 aprile 2007. 
